\title{
Protée
}

\section{Laïcisation de la littérature québécoise et tradition rhétorique. Un cours de littérature à l'École normale de Chicoutimi (1912-1913)}

\section{Fernand Roy}

Volume 28, numéro 1, 2000

Variations sur l'origine

URI : https://id.erudit.org/iderudit/030586ar

DOI : https://doi.org/10.7202/030586ar

Aller au sommaire du numéro

Éditeur(s)

Département des arts et lettres - Université du Québec à Chicoutimi

ISSN

0300-3523 (imprimé)

1708-2307 (numérique)

Découvrir la revue

Citer cet article

Roy, F. (2000). Laïcisation de la littérature québécoise et tradition rhétorique. Un cours de littérature à l'École normale de Chicoutimi (1912-1913). Protée, 28(1), 94-104. https://doi.org/10.7202/030586ar
Résumé de l'article

Cet article présente puis commente la théorie inhérente à un cours de littérature donné en 1912-1913 à l'École normale de Chicoutimi par un ex-professeur de Rhétorique. L'auteur y met en évidence que la croyance religieuse du professeur y sert d'explication justificative de la vision monologique du langage - et de la dyade " fond / forme " - que véhiculait, depuis Aristote, la tradition rhétorique. Et il propose que la laïcisation de la littérature québécoise ne se serait pas faite en réaction à l'idéologie cléricalo-religieuse, mais en continuant de cautionner une vision pré-dialectique du langage. 


\title{
LAÏCISATION DE LA LITTÉRATURE QUÉBÉCOISE ET TRADITION RHÉTORIQUE
}

\author{
Un Cours de littérature À L'ÉCole noRm ALe de Chicoutim I (1912-1913)
}

FERNAND ROY

[... ] nous défions qu'on puisse fournir effectivement une explication matérialiste des effets esthétiques littéraires sans mettreen évidence leur détermination internepar l'histoire de la langue national e et de l'appareil scolaire.

(E. Balibar et M. M acherey, «Présentation » des Françaisfictifs de R. Balibar, Paris, H achette, 1974, p. 15; le romain est de moi)

D ostoïevski ne garde jamais pour lui d'excédent interprétatif essentiel, mais seulement un excédent pragmatique minimal, informatif, nécessaireà la conduitedu récit. L'excédent interprétatif transformerait, en effet, legrand dialogue du roman en un dialogue objectif et achevé, en un jeu rhétorique.

(M . Bakhtine, La Poétique de D ostö evski, Paris, Seuil, 1970, p.114; le romain est de moi)

Soixante-cinq pages de «N otes [dactylographiées d'un] cours de littérature» donné à l'École normale (des filles) de Chicoutimi à l'automne 1912 et à l'hiver 19131. D ans un premier temps, ce «signe» m'avait semblédigned'intérêt pour diverses rai sons de stratégie de recherche2. Puis, comme cela arrive souvent quand jetravaille, je n'ai plus su qui, du sociologuede la littérature ou du sémioticien, était leplusintéressé. Cependant une évidence me sautait en effet aux yeux: les trente premières pages deces «N otes» val aient le déplacement, ellestémoignai ent d'un effort didactiqued'une qualitéremarquable et $m$ 'incitai ent à revoir l'idée - plutôt reçuequefondée objectivement - quej'avais del'idéologie ${ }^{3}$ cléricalo-religieuse dite de conservation alors dominante dans notre institution littéraire encore un peu à la recherche de sa spécificité. L'auteur, un ex-titulaire de la classe de Rhétorique du Séminaire deChicoutimi, y explicite, àl'intention de futures enseignantes, ce qu'est la littérature et pourquoi c'est essentiel pour elles de le savoir pour enseigner (entre autres matières) la languematernelleàl'écoleprimaire 4 , dans les écoles publiques.
Je voudrais rendre compte ici de ce que les «N otes» du professeur $D$ égagné $m$ 'ont aidé à comprendre de la vision de la littérature - et de son rapport au langage - véhiculée dans cetteÉcolenormale defilles au début du siècle. Comme comprendrele «signe» que constituent ces «N otes» implique d'interpréter celui-ci dans une perspective donnée, il me paraît honnête de commencer par exposer brièvement, sous forme de problématique et d'hypothèse à étayer ensuite, la lecture quej'en ai faite. Je formulerai cette lecture dans la proposition suivante: la rhétorique classique a une fonction surdéterminante dans la définition de la littérature proposée à des normaliennes en 1912-1913; cettefonction était surdéterminante en ce sens que l'idéologie cléricalo-religieuse qui recouvrait cette définition constituait une explication justificative à saveur métaphysique - construite après coup, à la fin du M oyen Âge - de la compréhension prédialogiquedu langage véhiculée par la tradition rhétorique.

\section{Problématique et hypothèse de travail}

Pour les fins du présent essai, je considère que la laïcisation de la littérature québécoise a été effective quand, audelà de la subordination du savoir écrire à la gestion du rapport entreles humainset l'au-delà, les littéraires ont fondé sur des études à saveur esthético-nationaliste la légitimation des textes déjà autonomisés- textes auxquels la critique avait suggéré de reconnaître une plus-value, au-delà de leur efficacité discursive immédiate et qui, à quelques exceptions près ${ }^{5}$, avaient déjà été intégrés dans le corpus officiel par les premiershistoriens del'institution. Pour comprendre le passage du «patrimoine sacré de la langue française» - l'expression est del'historien Camille Roy - au «corpus littéraire québécois» que nous véhiculons aujourd'hui, il s'avère fondamental de prendre aussi en compte le contexte immédiat dans lequel la littérature française ${ }^{6}$ a été présentée au début $d u X X e$ siècle à des futures institutrices du système d'en- 
seignement public. En effet, cesfuturesinstitutrices n'étaient pas tenues d'assimiler des rudiments de culture gréco-latine, ni tenues d'acquérir de formation philosophico-religieuse; elles dérogeaient singulièrement à l'idéal attendu de l'élite intellectuellequi pouvait avoir charge d'âme. L'ex-professeur de séminaire allait forcément devoir faire preuve d'imagination pour arriver à articuler, à leur intention, la philosophie chrétienneaux valeurs esthétiques dites «classiques»: habituellement, ces valeurs étaient implicitement justifiées, par le fait que la langue française avait des racines grécolatines qui garantissaient sa pérennité - et, partant, toute la tradition rhétorique dont on s'inspirait pour définir la littérature, dont l'art oratoireétait postuléle plus pur fleuron. $\mathrm{Ce}$ «contexte» nouveau allait d'ailleurs être l'occasion de la cristallisation de l'idéologie de la langue gardienne de la foi ; les romans historiques de L aureC onan y ont largement contribué en liant la langue française à la survie de la foi chrétienne?.

II me parait difficile de comprendre la laïcisation de notre littérature- valorisation del'érotismeet engagement dansle milieu à l'appui 8 - sans faire l'hypothèse que l'on s'est également interrogé au sujet de la pertinence de la tradition rhétorique pour rendre compte du langage. $D$ e deux choses l'une, ou bien le «savoir au sujet du langage» véhiculépar la tradition universitaire demeurait récupérable par la modernité, ou bien il fallait accepter de se demander froidement, avec D enis Saint-J acques ou autrement, si lesétudes de lettres relèvent de la supercherie ${ }^{9}$. En inscrivant explicitement la littérature dans le processus d'apprentissage de l'écriture, les «N otes» du cours de $D$ égagné m'ont amené à faire l'hypothèse quele soupçon desupercherieévoquépar Saint-J acques gagneà être débité explicitement du côtédel'impassequ'il y a à prétendre sauver la tradition rhétorique au moyen de la linguistiquestructurale. La doublearticulation des signes linguistiques ne peut être comprise que comme résultanted'un processusinteractif entre desinterlocuteurs qui collaborent dans le temps et dansl'espace en direction d'objets.

L'enseignement de l'«art oratoire» a, dans notre civilisation, été à l'origine de la réflexion sur le fonctionnement du langage. Cet art est né en «contexte judiciaire»: à un moment donné, il est apparu qu'il serait humainement plus opérationnel de savoir convaincre des interlocuteurs-juges, plutôt que de donner raison à ceux qui savaient imposer leur point de vue par les armes10. L'art de manier les mots a remplacé l'art de manier les armes. Par la suite, l'écriture permettant de prendre un recul critique à l'égard des divers usages langagiers 11 , Aristote a fait un lien entre les figures de rhétorique- liéesà l'art de convaincre desjugesà l'occasion des chicanes juridiques - et les figures de style - liées à la réception, après coup, des signes présumés moins mimétiques du monde (extérieur) qu'expressifs de l'âme (intérieur). Ce n'est pas manquer de respect pour A ristoteet pour la tradition rhétorique, que le Groupe $\mu^{12} s^{\prime}$ est appliqué à général iser, que d'affirmer que l'habitude d'interpréter les discours en distinguant entre le fond et la forme renvoie à une stratégie didactique de production d'argumentations: il s'était avéré efficace, pour arriver à convaincre un interlocuteur-juge, de commencer par s'occuper du fond (la délimitation du but à atteindre, puis l'inventaire, le choix et la mise en ordre des arguments les plus valables pour atteindre ce but) et de se préoccuper ensuite de l'interlocuteur même lors de la mise en forme (la composition proprement dite).

Le lien pressenti par Aristote entre les procédés des rhéteurs et ceux des poètes a eu comme effet d'occulter que c'est la situation de reproduction du langage- dont participe l'écriture - qui légitimise l'autonomisation de la forme, en l'occurrence du remplacement du maître-rhéteur par le maitre d'écriture. Q uand prévaut un objectif de reproduction d'usages langagiers déjà partagés, quand la finalité est d'amener des interlocuteurs-élèves à savoir faire preuve d'«éloquence», il devient évidemment utile de considérer que la langue est fonctionnellement autonome: on peut dès lors postuler que le cheminement del'interlocuteur-élève va consister à refaire le cheminement du locuteur-professeur. D elà à vouloir qu'il soit possible, pour un interlocuteur qui partage la même langue que le locuteur, de refaire - idéalement sans reste - le cheminement de ce dernier, il ne reste évidemment qu'un pas à franchir.

La situation d'apprentissage de l'écriture permettant d'oublier que des usagers qui interagissent occupent par définition des positions asymétriques, le «vase clos» permet de méconnaître - entre autres - 1) que dire d'un «signe» quec'est une «figure destyle» constitue à la lettre une hypothèse d'interprétation en fonction du contexte d'enseignement, c'est-à-dire à partir des seuls consensus acquis, constitutifs d'un état donné des usages, que le maîtreet les élèves sont supposés partager; 2) que, l'interprétation par un interlocuteur étant une composante du processus d'intercompréhension d'où résultent les usages langagiers, c'est en faisant abstraction des positions asymétriques desinterlocuteurs que I'on finit par imaginer qu'un interlocuteur peut refaire le cheminement d'un locuteur et circonscrire avec relativement 
de succès le sens - en langue - de ce qui sera dès lors une «figure de style» 13 ; et surtout 3) quel'objectivation desconditions d'existence d'un signe doit normalement conduire non pas à sa signification mais à la mise en évidence de la double articulation asymétrique qui le constitue justement comme «signe», donc comme «orientant de l'intérieur la relance qu'il appelle». D'où ma proposition au sujet de la fonction surdéterminante de la tradition rhétorique dans le processus de laïcisation de la littérature québécoise: la libéralisation del'institution littérai requébécoise aurait reconduit la «compréhension» rhétorique - pré-dialectique - du langage que justifiait l'idéologie de la langue gardienne de la foi.

La situation d'enseignement permettant de méconnaître que l'interprétation de l'interlocuteur est constitutive dans letemps et dans l'espace du lien entre le signe du locuteur et son objet, la laïcisation de notre littérature aurait été l'occasion d'un remplacement un peu à courtevue d'uneidéologie jugée passéiste par une idéologie dite moderne qui risquait de fonctionner simplement «à rebours»: de la censure à la val orisation de l'érotisme; de la quête de l'au-delà à la quête du pays d'ici. Fautede concevoir clairement qu'un consensus langagier résulte de l'amal game de points de vue différents en direction d'un objet à faire exister langagièrement, on se réacheminait vers une vision destextes littérairesqui risquait des'avérer tout aussi désincarnée quecellequel'on dénonçait. Ce risque constant de «structuralisme» me paraît lié à la déportation «monologique», du côté de la réception, de la dyade «fond / forme», qui n'était somme toute qu'une stratégie didactique. Concrètement, on confondral'objet du discours du maîtreà ses élèves avec l'objet du discours retenu comme modèle. L'élève sera le garant de la confusion, qui sera appelé, tel un présumé «hypocritelecteur», à cautionner en termes de symbolisme - plus ou moins romantique ou esthétisé ou naturalisé ou textualisé - qu'un texte littéraire serait un discours «spécial», qui lierait volontairement, mais pas seulement pour persuader, une «nébuleuse» - au sens de «surplus» - de «contenu » à une «forme d'expression» exceptionnelle14.

Jetenterai d'étayer cette hypothèse - de confusion entre deux objets 15 - à partir du commentaire d'un quatrain proposé par le professeur $D$ égagné à ses interlocutrices de 1912-1913 pour illustrer sa définition dela littérature. $M$ ais avant d'y venir, il convient de raconter le plus fidèlement possible la «théorie» de la littérature que voulait illustrer le commentaire en question.

\section{2) Le cours de littérature du professeur D égagné}

Jecommencepar la présentation du lieu, l'Écolenormale des filles de C hicoutimi, et des interlocuteurs en présence, le professeur et les futures enseignantes.

Les Sœurs du Bon-Pasteur avaient ouvert leur Écolenormale pour les filles en 1906, avec bien sûr l'aval des évêques, puis du Comité catholique du Conseil de l'instruction publique 16. Comme c'était l'habitude al ors, I'École des Sœurs du Bon-Pasteur avait sa propreécole d'application - les Sœurs du Bon-Pasteur avaient une école à $\mathrm{C}$ hicoutimi depuis une trentaine d'années. La finalité poursuivie par l'institution de formation était de procurer aux futures institutrices la compétence nécessaire à l'enseignement: d'une part, en complétant «leur formation intellectuelle, religieuse et morale»; d'autre part et surtout, en les initiant à «la science de l'enseignement et del'éducation ». I déal ement, les cours de complément de formation étaient orientés vers l'enseignement.

Confiées à des communautés de religieuses, les Écoles normales de filles étaient supervisées «administrativement» par un Principal nommé par les évêques. À Chicoutimi, de 1914 à 1940, le Principal a été le professeur du cours de littérature ici en cause, I'abbé $N$ arcisse $D$ égagné. O rdonné prêtre en 1889 17, M aître ès Arts de I'U niversité Laval en 1896, N arcisse D égagné s'était d'abord acquis une solide réputation au Séminaire de Chicoutimi, à titre de titulaire pendant dix-sept ans de la classe de Rhétorique, mais aussi à titre de directeur desétudes puis del'institution même. Indé pendamment de l'incendie de celle-ci, sa seconde carrière avait en un sens été préparée. Avant de devenir le Principal de l'École normale en 1914, il avait été le confesseur de ses religieuses; et il y avait aussi assuréquelques cours, dont celui de littérature, qu'il a donné pour la première fois- le soir, et à des religieuses - en 1912-1913. Je ne saurais dire avec certitude combien d'années $D$ égagnéa repris ce cours auprès des normaliennes. $D$ es recoupements m'amènent à penser qu'il l'a fait pendant plus de vingt ans: il publiait toujours des analyses de texte modèles dans la revue L'Enseignement primaire en 1934; après cette date, il a publié uniquement unechroniquedelangue dansleP rogrèsdu Saguenay, jusqu'en 194018 .

Au début des années vingt du siècle dernier, on se disait queles finissantes des Écoles normales defilles devaient avoir au moins dix-sept ans. Les certificats émis ensuite allèrent même jusqu'à l'attester. Ils précisaient aussi qu'elles étaient de religion catholique et quel'on n'avait rien remarquédans leur mœurs, conduite ou caractère qui puisse les rendre 
impropres à exercer la fonction d'institutrice. Bien sûr, ces certificats leur reconnaissaient égal ement, après deux ou trois ans dans les cas qui nous concernent, une compétence pour I'enseignement. Pour être admises à l'École normale, les jeunes filles, qui avaient déjà complétéles six années del'école publique d'alors, devaient réussir un examen d'admission. Par définition, elles étaient tenues d'être pensionnaires 19 . Avant 1920, le «C ours de littérature» de D égagné semble avoir toujours été donné à moins de vingt normaliennes, souvent à moins de dix.

Les «N otes» du coursqui nous sont parvenues sont divisées en dix-sept leçons que je qualifierai de théoriques, en ce qu'ellesnefont qu'occasionnellement référence aux écrivains, àl'histoire littéraire mêmeet à tout cequi concerne les devoirs usuels; il s'agit des compositions avant $\mathrm{N}$ oël et des analyses littéraires après 20 . C es dix-sept leçons ${ }^{21}$ explorent systématiquement ce que leur auteur identifie, chemin faisant, comme les trois conditions de l'art d'écrire: la connaissance despréceptes, l'étude des modèleset la pratiquedela composition. La partie «connaissance des préceptes», qui a surtout retenu mon attention, expose ce qu'est la littérature à partir de l'expression «bien parler». Avant d'y venir puis de la commenter, je résumerai en quelques mots ce qui concerne les deux autres conditions del'art d'écrire; neserait-ceque pour établir clairement que le professeur inscrit la littérature dans une perspectived'apprentissage del'écriture, d'apprentissage dela «paroleécrite» pour reprendreune expression qui rend bien tout ce que, sel on lui, l'art d'écrire doit àl'art oratoire22.

D ans un deuxième temps, du côté des «pratiques», le professeur suggèrequel'étude desmodè esse fait par la lecture, idéalement crayon en main, des grands maîtres ${ }^{23}$, par l'anaIyselittéraire, la traduction et l'imitation. L'analyse littéraire est définiecommeun exercice qui inverse le processus normal de la composition; concrètement, cela implique de circonscrire l'idée de fond du texte, d'en dégager l'organisation interne puis de rendre compte de la qualité du style, de la virtuosité avec laquelle l'écrivain a su lier l'expression au contenu ${ }^{24}$. La traduction est décrite commeuneactivitéqui fait travailler l'intelligence, l'imagination, l'âme et le goût: elleobligeà assimiler la pensée d'un auteur jusqu'àcequ'elle devienne celle du traducteur. Enfin, par imitation, il faut entendreun exerciceintelligent: unefois letexted'un maître bien assimilé, on en assumedefaçon personnellela réécriture, comme les classiques français ont fait avec les classiques anciens, latins et grecs! Bref, l'étude des modèles donnetoute sa portée au terme «classique», utilisé par le professeur en un sens qu'il dit premier, à savoir «qui mérite de servir de modèles dans les classes» 25 .

Comme le veut la tradition, quand on applique la rhétoriqueà la cultureécrite ${ }^{26}$, la composition comportetrois phases successives: I'invention, la disposition et l'élocution 27. La troisième, l'élocution, étapedel'écritureproprement dite, n'est pas tellement présentée de façon théorique 28 . Cet enseignement a été intégré - le cahier manuscrit de préparation immédiate en témoigne largement - lors de la pré paration et de la correction des devoirs 29 . En tout cas, les «N otes» dactylographiées ne font pas explicitement référence aux quatre parties que l'on sait: I'exorde, la narration, l'argumentation et la péroraison 30 . Par contre, les deux phases préparatoires qui doivent précéder I'«élocution » sont l'objet d'une attention théorique toute particulière. Sans parler explicitement des «topiques» de la logique aristotélicienne, le professeur propose des «trucs pratiques» incontournables, des instruments infaillibles qu'il nomme «lieux communs»: la définition, l'énumération des parties, la causalité, les circonstances et les rapports de ressemblance et de différence entre les choses, mais aussi - car il ne faut tout de même pas confondre la finalité sacrée et les écrits profanes d'A ristoteentre les créatures et le $\mathrm{C}$ réateur. $\mathrm{Q}$ uant au choix (toujours difficile) de ce que l'on va conserver ensuite et à l'ordonnancement des idées en grâce, en force et en beauté en fonction de la finalité à poursuivre, à propos de la «disposition » donc, voici un passage représentatif et du ton de l'ensemble des «N otes» et de ce que le propos du professeur «adapte» la tradition née de l'art oratoire:

II ne faut retenir que ce qui conduit au but. [...] L'avocat devra serrer son sujet, ne pas oublier un instant le but qu'il se propose; le poète, le conteur sont pluslibres I Issont pluslibres, mais il ne doivent jamais écrire selon le caprice, la fantaise. Dans le caprice, il n'y a pas de littérature, il n'y a rien qui éève les âmes. Le poète écrit par le seul amour de l'art, sans intérêt matériel ; il et donc plus coupable quand il corrompt lesâmes. 31

II faut choisir parce qu'il faut álever les âmes, les faire tendre vers le bien. Bossuet ordonne ses écrits avec un grand soin, le contraire existechez Victor H ugo. D ansBalzac cen'es plus de l'ordre, ni même du désordre ordinaire, ce sont de véritables vues ani mées [...] vues basses et réalistes (p. 53-54)

En somme, un art de la «parole écrite» qui affuble l'écrivain d'unemotivation sansintérêt matériel, l'amour del'art, et qui lui reconnaît une certaine liberté dans les moyens, 
unelibertéqui toutefoiss'avèrebien délicateà gérer, puisque par définition elle a comme finalité l'au-delà de la vie. Le tout seterminesur une idée-synthèsequi, rhétoriqueoblige, veut laisser une impression forteaux normaliennes: la parole de l'artiste, et même celle de l'artisan, doit être idéaliste; «dans le matériel, [elle se doit de considérer] l'immatériel; dans la beauté finie, la Beauté infinie»32. C'est là une reconfiguration, en fin deparcours, de la définition proposée au point de départ et développée dans la première partie du cours.

Lestoutes premières phrases des «N otes» méritent d'être lues attentivement, avec toute l'empathie dont on peut être capable. Au-delà des apparences, c'est-à-dire de l'apparente simplicité du propos, Dégagné y a mis, je trouve, tout son doigté:

La littérature, c'est l'art de bien parler... Bien parler suppose quel'on a bien pensé, bien senti... pour ensuite bien écrire. La littérature, c'est donc l'art d'écrire, et de plus de juger les écrits des autres, 33

Q u'es-ce que bien parler? Bien faire une chose quelconque, c'est la faire conformément à sa natureet à sa fin. C'est mettre tout son talent, tous les soins possibles pour atteindre la fin, arriver au but quel'on se propose. Bien parler, c'est donc parler de la manière la plus conforme à la nature et à la fin de la parole. M ais qu'est-ce que la parole? II y a deux choses dans toute paroleécriteou parlée: 1) un objet exprimé, 2) uneâme. (p. 1)

La différence entre la «parole» du commun des mortels et celle d'un écrivain qui a du talent - ou mieux, du génietient au «bien» dans «bien parler». Ce «bien » présume, les «N otes» en conviennent ouvertement, que le commun des mortels ne parle qu'à moitié, faute d'avoir du «génie». Ce «bien » signifieen l'occurrence: «en mettant en œuvretoutes les facultés del'âmeet en respectant leur hiérarchie naturelle». Avoir bien senti d'abord et bien parler ensuite, c'est le faire avec toutes les facultés de l'âme humaine et en respectant la hiérarchie naturelle de ces facultés. En explicitant cela, D égagnéen vient à soutenir que, sommetoute, quand l'âme y est tout entière, les mots ne sont qu'unesorte devoileposé sur les choses - ce serait cela une parole pleine, j'imagine, une parole qui dirait l'immatériel, une parole à «objet exprimé» transcendé. Avoir bien senti puis parler avec toute son âme permet d'accéder au vrai, au bien et au beau; ce vrai, ce bien et ce beau, cela D égagnéle sait par l'Écriture34, sont le reflet de Dieu dans sa création - dont l'écrivain participe, alors que le commun des mortels ne sent et ne parle qu'à moitié, qui, si j'ai bien compris, s'en tient à la partie «objet exprimé», sans parvenir à «l'âme».

Bien sûr, jebrûle ici lesétapes. D égagnépasse sept leçons à expliciter sa définition formulée au point de départ. $0 \mathrm{n}$ l'a vu en parlant de la disposition: selon lui, l'art commence au-delà des «objets exprimés» simplement dans un but utilitaire; un peu comme la mise en forme intervient une fois que le fond est arrêté dans la stratégie des premiers rhéteurs, mais à une différence près, qui est capitale: il s'agit cette fois de parler vrai, «comme pourrait le faire D ieu »- et non pas seulement comme pourrait le faire un interlocuteurjuge reconnu comme particulièrement sage par l'ensemble de la communauté et qu'il s'agirait de convaincre35.

La deuxième leçon expose que les facultés constitutives de l'âme sont globalement, d'une part, l'intelligence et la volonté et, d'autre part, l'imagination et la sensibilité- à ne pas confondre avec les sens. Elle circonscrit la spécificité de chacunede cesfacultés. Exemple: I'intelligence est la première de nos facultés; son rôle est de comprendre, et comprendre c'est accéder au vrai, l'embrasser; elle n'y arrive pas par l'intuition, comme peuvent le faire les anges qui ont le privilège de «voir » $D$ ieu directement, mais bien par ledétour de la raison 36 . Tel est le lot des descendants des premiers humains: ils ont une intelligence qui n'accède au vrai, au beau et au bien qu'en travaillant intellectuellement. Exemple encore: l'imagination est une facultéqui fait le lien entreles sens et l'intelligence; ou entrel'intelligence et les sens, puisque l'âme forme, depuis la naissance jusqu'à la mort, un tout avec le corps. Sur la base de cette psychologie de l'âme humaine, $D$ égagné formule ensuite sa thèse centrale: la force d'un écrivain tient àcequ'il sait, par son imagination, rendre sensible aux autres âmes la beauté dont son âme particulièrement sensible a eu l'intuition. En clair: ne jamais oublier que la justice humaine - dont participe la rhétorique - est un bien faible écho de la justice divine.

La formule qui lui était chère- l'art est l'expression sensible du beau immatériel - cautionnait le précepte de la hiérarchie des facultés de l'âme et permettait d'expliquer la supériorité des classiques: les romantiques avaient malencontreusement eu tendanceà insister trop exclusivement sur la beauté sensible, celledel'objet exprimé. Q uant aux tenants del'art pour l'art et autres naturalistes, ils auraient, àl'inverse, sombré dans un orgueil intellectualiste mal heureux: ils ont ignorél'absurditéqu'il y aà soutenir qu'une partiedel'activité humaine - celle de l'âme - échappe à sa finalité naturelle, à 
savoir Dieu dont naturellement elle se doit de témoigner. On l'aura entendu: savoir amal gamer correctement les objets et l'âme n'est hélas! pas à la portée de tous les talents! Je résume. «Bien parler», c'est exprimer la vérité des choses, le reflet de $D$ ieu dans les choses; bien parler implique d'exprimer cette véritéavec force, c'est-à-dire avec toutes les facultés de son âme, pour en convaincre les âmes de ceux qui sont moins favorisés, qui ont peu de talent.

Je termine cette narration orientée sur un des rares exemples contenu dans les «N otes» de D égagné, exemple qui illustreà la fois sa compréhension du langage (fond, puis forme) et le respect de la hiérarchie des facultés de l'âme dont il se sert pour justifier cette compréhension:

$\mathrm{T} e \mathrm{en}$ un secret vallon,

Sur lebord del'onde pure

Croît à l'abri de l'aquilon

Un jeunelis, l'amour de la nature. (Racine ${ }^{37}$ )

Premièrement l'idée: c'est l'innocence qui vit à l'abri de tous les dangers. D euxièmement l'image symbolique: l'image est quadruple: lis, mis pour vous [les normaliennes, bien sûr]; vallon, le couvent; onde pure, la vie innocente; aquilon, les dangers. Sentiment de douceur, de joie pure, de bonheur pur et vrai, letout admirablement fondu dansla même phrase $\mathrm{Ce}$ qui fait la beauté d'un ouvrage littéraire, c'est la verrité des idées, la beautéet lenaturel desimages, la chaleur du sentiment. C'est une pensée haute, des sentiments nobles, purs, exprimés simplement Si le sentiment est noble, élevé, pur, l'expression sera demême. N'ayez quedes sentiments nobles élevés, et vous écrirez bien. (p. 9$)^{38}$

\section{Commentaire critique}

D 'abord deux détails relevant de la mise en page - de I'énonciation-, qui sont révélateurs de la compréhension «symétrique» du langage que ce commentaire actualise: le «vous» qui pointe explicitement les destinataires (interlocutrices) est soulignéau mêmetitrequeles mots «lis», «vallon» «ondepure» et «aquilon», qui constituent, selon lescripteur, une «image symbolique» «quadruple»; et le mot «lis» a par la même occasion été ramené avant les trois autres, qui le précèdent pourtant dans le petit quatrain. Le premier détail renvoie explicitement au précepte selon lequel l'art doit servir à toucher l'âme: un «sentiment [...] noble, élevé, pur » y parle à l'âme des interlocutrices, la touche dans son aspiration à l'immatériel qu'elle a en commun avec celle (plus sensibleet plus imaginative) du talentueux poète. En écrivant «lis, mis pour vous, [...] 》, D égagné abolit la différence entre «vous» en tant que lectrices de Racine et «vous» en tant que cellesà qui il s'adresse; tant et si bien qu'est dès lors assurée une équivalence, du côté de l'objet dont il est question dans le poème, entre les deux signes que les «vous» «reçoivent». Cette équivalence rejoint l'idéal detransparence, l'impression de parole comme simple voile déposé sur les choses et, surtout, la croyance selon laquelle un lecteur peut refaire le cheminement d'un scripteur: «Premièrement l'idée: c'est l'innocence [...] Deuxièmement I'image symbolique: I'image symbolique est quadruple».

La petite strophe invite indéniablement à «voir» une manifestation d'amour - divin selon le poète - dans le fait d'un jeune lis donné, qui croît à l'abri de l'aquilon sur le bord de l'onde pure 39 . À la condition, bien évidemment, de passer sous silence qu'en rêvant de la sorte on fait comme si «un paradigme déjà là» était annoncé dans «T el en un secret vallon » puis rabattu sur «le syntagme» «l'amour de la nature». En pratique, c'est plutôt «le syntagme» «l'amour de la nature» qui, au-delà et seulement au-delà de «Sur le bord de l'onde pure / C roît à l'abri de l'aquilon / U n jeune lis», permet de «voir» «un paradigme» dans «Tel en un secret valIon ». La prétention du professeur de refaire le cheminement du poète lui permet d'occulter qu'il n'est pas en interaction avec ce dernier mais bien avec des interlocutrices, à propos de quatre vers; cette méconnaissance l'amène à croire que le quatrain fait du sens «tout seul »- en direction de l'infini.

$M$ on hypothèse est à l'effet que la tradition rhétorique permet d'occulter le discours du maître. Et de postuler, à l'occasion d'une «parole» - le petit quatrain, en l'occurrence - qu'il existe quelque part de la «forme langagière en soi »: en reconduisant, pour fin de compréhension d'un signe déjà construit, la dyade «fond / forme» constitutive de la stratégie imaginée pour produire un discours efficace, le professeur postule la transparence de la forme, dont la croyance des romantiques au symbole n'est qu'une variante, à l'aube de notre modernité40. D epuis leXIX ${ }^{\mathrm{e}}$ siècle, le symbolisme littéraireaidant, on fait commesi cela existait tout seul, de la forme. Il est en un sens paradoxal que, tout en se servant luimêmedelanotion desymbole, D égagnéait dénoncél'orgueil «intellectualiste» des successeurs des romantiques que sont les tenants de l'art pour l'art et les naturalistes. Le paradoxe n'est cependant qu'apparent: les explications par la théologie et par l'esthétiquene font quejustifier demanière différente l'idée que «de la forme» cela existe en soi, idée qui nous vient du monologisme inhérent à la tradition rhétorique ${ }^{41}$, 
qui prend une stratégie didactique pour un savoir au sujet du fonctionnement du langage.

La «reconnaissance» - en contexte scolaire, de reproduction du langage- d'une «métaphore» dans «C ettefemme est une fleur » peut ici faciliter la compréhension en divisant la difficulté. Le discours à décrire y devient une «forme d'expression »à interpréter en fonction d'une culture langagière déjà partagée. En pratique, le professeur - que l'on peut présumer créateur de «C ette femme est une fleur» adresse à ses interlocuteurs la proposition suivante: «"C ette femme est une fleur" est une métaphore». Pour vérifier son hypothèse de «figure de style», il réduit alors l'allotropie perceptibledans le discours donnéen mettant en rapport les composantes sémantiques de «femme» et de «fleur», jusqu'à identifier des traits communs possibles. Cette pratique lui permet demettreen évidencediverses possi ibilités decompréhension. C ela ne prouve pas que «C ettefemmeest unefleur » est une métaphore; cela illustre tout juste l'asymétrie de la doublearticulation du discoursqu'il s'est donnécommeobjet d'analyse. En y décelant une «métaphore», le professeur fait comme si les possibilités de compréhension en langue qu'il évoque préexistaient à son propre discours; il occulte son geste interprétatif en le présentant comme vérification de ce que «C ette femme est une fleur » serait une parole spéciale, métaphorique. Le professeur ne prend alors pas acte que sa «création discursive» n'est pas forme d'expression en soi, mais bien pour un interlocuteur. C'est uniquement en contextescolaire de reproduction du langage que les métaphores existent! Elles servent à illustrer le fonctionnement des discours, la double articulation qui en fait, en direction des autres et par eux, des signes.

D evant la petite phrase moinslinéaire attribuéeà Racine, on n'arrive pas à oublier aussi facilement la discursivité qui la constitue. C e ne serait pas «C ettefemme» mais bien «T el en un secret vallon» appelant «l'amour de la nature» qui constituerait l'«aboutissant» dont le «tenant» serait «Sur le bord de l'onde pure / C roît à l'abri de l'aquilon / U n jeune lis». Le simple bon sens oblige à prendre acte que «Sur le bord de l'onde pure [...]» suit «Tel en un secret vallon», mais précède «l'amour delanature». En déplaçant laquestion du plan del'énoncéà celui del'énonciation, en postmoderne astucieux et averti, E co a suggéré qu'il faut circonscrire la spécificitédu symbolismelittéraire du côtédelamiseen discours: lemodesymboliqueaurait comme spécificité la mise en place d'une «forme d'expression exceptionnelle»à laquelleserait liéeune «nébuleuse» - au sens de surplus et non pas d'indétermination - «de contenu». Voyons voir où peut mener ce tacite maintien de l'esprit monologique de la dyade «fond / forme».

Ce que $D$ égagné reconnaissait comme «fondu dans une même phrase» relève effectivement d'une stratégie de mise en discours. La difficulté tient dès lors à ce qu'il faudrait ne pas annuler le rôle de la versification - en termes de transparence ou autrement - sous prétexte qu'en soi les rimes ne «veulent rien dire». II en irait en quelque sorte d'un équivalent du second niveau d'articulation - celui des phonèmes dont a parlé $M$ artinet dans une perspective de linguistique structural $\mathrm{e}^{42}$. Letraitement «prosodique» des deux isotopies déjà sémantiques, et par ailleurs enchâssées detelle sorte qu'il s'avère nécessaire de prendreen comptela discursivitéqui les constitue, impose pourtant un second découpage des unités «déjàsignifiantes»: lerejet du sujet «U n jeunelis» del'isotopie enchâssée vers le quatrième vers a comme effet d'en fairetout spécialement un complémentaire possible de «l'amour de la nature». À ce «plus» que pointe Eco, on ajoutera aussi que la reprise dans «aquilon» de la sonorité marquée à la fin du premier vers, dans «vallon», se double de la reprise dans «nature» de la sonorité finale - »pure» - du second vers. II ne me semble pas qu'il faille, pour autant, parler d'une «nébuleuse de contenu»: j'y décèle tout au plus des contraintes supplémentaires à prendre en compte pour entendre cequele scripteur voulait - au sens de «essayait de»- suggérer. Plutôt que de parler, par conséquent, de limites de l'interprétation, je suggérerai donc l'idée de «balises d'interprétation » que le scripteur a ajoutées pour orienter le travail de lecture.

Pour circonscrirel'effet escomptéde ces balisesformelles, je trouve opérationnel de recourir aux relations de présupposition, de complémentarité et de contradiction définies par les hjelmsléviens. 0 n constate alors que le petit quatrain amalgame la temporalité inhérente à l'enchâssement syntaxique et la temporalité inhérente au croisement des rimes, jusqu'à baliser une double relation de présupposition dont le second volet prend temporellement appui sur le premier. Comme ceci, en décalant le second volet:

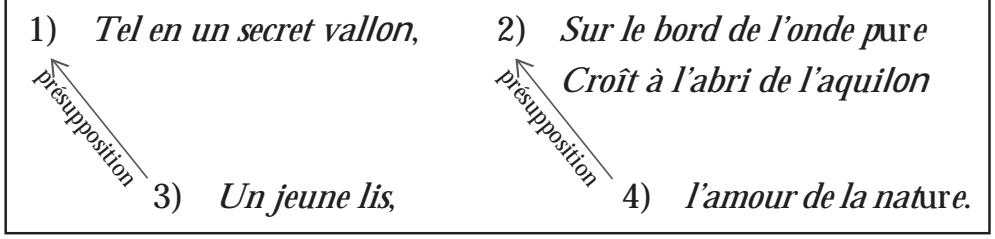


Ce petit schéma - un avatar de carré sémiotique... qui aurait une composante temporelle et qu'il conviendrait d'entendre commestructureélémentaire designifianceplutôt que de signification - veut illustrer les contraintes de lecture supplémentaires, liées à la versification, qu'un lecteur du quatrain se devrait de prendre en charge au-delà des usages, en langue, déà convenus:

- «U n jeune lis»n'implique «T el en un secret vallon» quesi «Sur le bord de l'onde pure / Croît à l'abri de l'aquilon» n'est pas lelot de tous les jeunes lis- que s'il n'est pas dansla nature d'«Un jeune lis» de croître à l'abri de l'aquilon sur le bord de l'onde pure;

- «un amour de la nature» n'impliquera «Sur le bord de I'onde pure / C roît à l'abri de l'aquilon» que si, par conséquent, «T el en un secret vallon » n'est pas, de quelquefaçon, toujours le lot de «l'amour de la nature» - que s'il est des «intellects» pour qui l'amour de la nature n'est pas inéluctablement «T el en un secret vallon».

En d'autres termes: la mise en «forme» imaginée par les premiers rhéteurs pour adapter le «fond » d'un discours à un interlocuteur-juge gagnerait effectivement à être entendue comme modalité discursive: elle n'aurait cependant rien à voir avec le «plan du contenu», du moins si j'ai raison de prétendre que dans le petit quatrain ici en cause la versification n'est pas porteuse de sens.

\section{En guise de conclusion}

Dans un processus d'intercompréhension, les interlocuteursen présence participent tous les deux à la construction d'un «objet langagier». T ous les deux participent de façon asymétrique à convenir du sens - en «langue» -, à reconnaître, disons, à l'expression «relever le gant»; et tous les deux conviennent égal ement, dans le temps, du défi précis à relever - en «contexte». Si ma lecture du commentaire du professeur D égagné est sémiotiquement opérationnelle; si j'ai raison defairel'hypothèseque dansle petit quatrain qu'il commentait laversification est del'ordred'un premier lecteur imaginaire et particulièrement complaisant qui insiste pour amener le lecteur réel à comprendreceque lescripteur voulait diresans déformer son intention; alors, il meparaît plausible de conclure que la difficulté de la tradition rhétorique tient à ce qu'elle a malencontreusement méconnu la composante temporelle inhérenteà toute mise en discours. Avec comme conséquencequela meilleure bonnevolontédu mondepeut conduire encore aujourd'hui à imaginer, avec U mberto Eco ou autrement, des contenus supplémentaires nébul eux à des formes d'expression finement élaborées, mais qui n'ont finalement pas d'autrevertu quecelle detabler - notamment à l'écrit - sur l'absence d'interlocuteur pour baliser subrepticement les contraintes delecturequetout interprètese devra de respecter pour comprendre. Si mon hypothèse est valable, le petit quatrain commenté par D égagné n'ajoute pas tout seul un supplément de contenu à «U n jeune lis»; la preuve ultime en serait que $D$ égagné avait besoin du «vous» de ses interlocutrices pour prétendre que c'était le cas: immé diatement après avoir affirmé l'idée, soit «c'est l'innocence qui vit à l'abri de tous les dangers», il ajoutait «lis, mis pour vous».

Un peu de la même manière, ce serait par le biais d'un symbolisme évanescent que la littérature québécoise a été libéralisée; en remplaçant l'idéologie de la langue gardienne delafoi par une idéologieesthético-nationaliste- valorisation de l'érotisme et engagement social à l'appui. Avec comme résultat la tendance des professeur(e)s à amener les étudiant(e)s en lettres à se prendre pour l'objet des textes qu'ils commentaient, plutôt que de les amener à réfléchir sur le fonctionnement dialogique du langage.

\section{NOTES}

1. «N otes du cours de littérature donné par M onsieur l'abbé $N$. D égagné à l'École normale de Chicoutimi 1912-1913», Chicoutimi, Archives de la maison provinciale des Sœurs du Bon-Pasteur, 65 feuillets (texte dactylographié). Lesréférencesàcedocument seront inscrites entre parenthèses directement dans le texte.

2. À ma connaissance, les recherches socio-historiques portant sur l'enseignement de la littérature n'ont jamais réussi à prendre en compte le fait que la pratique des textes «littéraires» s'inscrit d'abord dans le processus de scolarisation: on a toujours spontanément tendance à parler des réseaux de production des corpus, à privilégier le mouvement desidées ou à pointer les luttes de pouvoir dans le champ culturel, sans jamais réussir vraiment à subordonner de façon opérationnelle ces corpus, ces idées ou ces luttes à ce qui les caractérise en premièreinstance, soit la fonction del'appareil scolaire dans l'organisation d'une société dite démocratique.

3. Uneidéologie est une rationalisation qui à mon avisjustifie un discours posé comme savoir au sujet du langage. C en'est ni cohérent ni incohérent, cela permet, tant bien quemal, de «faireavec » unefaçon depenser lelangage qui, autrement, prendrait l'eau. Commeon nepeut pas penser sans postuler que l'on pense le langage correctement, on invente des explications pour 
justifier notre compréhension - partielle - du langage. L'idéologie, cen'est pas la compréhension, si partielle soit-elle, mais bien l'explication imaginée pour en colmater les brèches.

4. Avant 1923, l'école que nous disons aujourd'hui primaire comportait quatreannées d'étude; cetteformation général eétait possiblement suiviede deux années dans une école dite modèle. Après 1923, le primaire allait comporter six années et déboucher sur deux années complémentaires de formation «professionnelle».

5. À ce propos, il est à noter que la justification par les historiens de I'intégration tardive, après la réédition «amendée» de 1946, de M arie Calumet mis à I'Index en 1904, demeure symptomatique: les historiens de lalittératureont dansun premier tempscontinuédereconduirelejugement esthétique défavorable qui avait accompagnésa miseàl'I ndex, allant même jusqu'à dire que le seul mérite du petit roman en soi «sans consistance» de Girard tenait dans le fait que sa mise à l'Index constituait un événement digned'êtreconsignéparcequerévélateur del'étroitessed'esprit descenseurs d'avant la libéralisation de l'institution littéraire. Louise M ilot, François 0 uellet et moi-mêmeavonsmontréquelepetit textedeG irard constitueun récit fort bien organisé et aussi consistant que les autres romans par ailleurs déjà reçus comme «bien écrits» («L'inscription de l'écriture dans $M$ arie Calumet», Voix et images, vol. 10, $\left.n^{0} 1, p .80-95\right)$. J'ai également résuméles étapes de l'intégration de ce roman au corpus littéraire québécois dans l'introduction intitulée«M arieC alumet etl'institution littéraire»del'édition de la Bibliothèque québécoise (M ontréal, 1990) rééditée trois fois depuis. On peut faire le même constat à propos des D emi-civilisés d'H arvey, le dernier roman à avoir été mis à l'Index par un évêque au Q uébec: coincé entre le jugement esthétique négatif redoublant la condamnation lors de la mise à I'Index et l'importance de l'événement à consigner, les historiens littéraires des années soixante ont pris le parti de reconduire le jugement esthétique négatif tout en soulignant l'importance de l'événement dans le cheminement conduisant à la libéralisation de l'institution littéraire. II faudra bien un jour que quelqu'un prenne le temps de démontrer que I'institution littérairequébécoisea eu tendance, au moment desa laïcisation, à continuer de déprécier les textes qui épousaient de trop près le style journalistique: une fois laïcisées, les valeurs esthétiques se devaient tout de même de continuer à avoir de la classe, à ne pas trop ressembler aux usages de l'écriture pour fins de communication journalistique.

6. À toutes fins pratiques, entendre, du moins dans le cas ici considéré, d'abord les classiques français du XVIIe siècle

7. La pérennité de la langue française n'étant plus assurée par le seul apprentissage du grec et du latin qui permettait de participer de la tradition aristotélicienne, il devenait nécessaire de donner une finalitéstable (divine) à notre histoire

8. Les thématiques de «libération sexuelle» et d'«engagement social» communes aux écrivains de la génération de l'H exagone seraient nées d'un projet (poétique) de réconciliation avec la vieet d'acceptation du milieu qui seserait cristalliséen liant érotismeet quêtedu pays. C'est en tout cascequ'a dégagé Richard Giguère dans son enquête sur «La réception critique de I'H exagone dans les revues, 1954-1970», publié dans R. Giguère (dir.), Réception critique des textes littérai res québécois, Sherbrooke, D ép. d'études françaises, 1982, p. 149-197. D 'où la thématique dela femmecommeobjet d'uneconvention entredes hommes en quêtedepays; cequ'asi subtilement dénoncé Suzanne Lamy, au début des années quatre-vingt, en écrivant La Convention (VLB, 1985), où ellefait coller danslejournal deson héroïnedes lettresd'amour deson amoureux qui avait voulu - encoreen 1976 - lui faire I'amour en pleine nature «montréalaise», ce qu'elle lui avait sur le moment refusé parce que cela la gênait un peu, devant tout le monde... D e son côté, Patricia Smart témoignait de la difficulté d'écrire dans la maison du père (Écrire dans la maison du père: I'émergence du féminin dans la tradition littéraire du Q uébec, M ontréal, Q uébec-Amérique, 1988).

9. D ans son magnifique article de 1970, («Les études de lettres: une supercherie?», Études littéraires, vol.3, n02, p. 222-243», Saint-J acques étayait la nécessité de rendre compte de la littérature dans une optique de reproduction du langage, mais il préférait (encore) essayer de conjuguer érotisme et engagement social en proposant defaire appel à la psychanalyse pour rendrecomptedel'utilitésocialedela production del'imaginaireécrit. O n ne tenait pas à passer pour des charlatans, mais on arrivait mal à définir le rôle des agents chargés de gérer la reproduction de l'imaginaire écrit.

10. J e prendrai ici le risque d'essayer de rappeler brièvement l'origine, dans notrecultureoccidentale, del'art oratoire. II y aenviron deux millesept cents ans, les citoyens de la ville de Syracuse ont été spoliés de leurs biens par des tyrans ayant à leur solde une armée de mercenaires. Au bout de «X $X$ années, ayant réussi à libérer leur ville, les citoyens ont fait le projet de rétablir la situation d'avant les tyrans. À cette fin, ils se sont entendus sur un mode de fonctionnement qui - on le comprendra - excluait I'usage de la force des armes; mode de fonctionnement que les historiens de notre culture considèrent à l'origine et de notre idéal de démocratie et de notre pratique de l'art oratoire. Ils se sont entendus sur un certain nombre de sages qu'ils ont nommésjuges; et ceux qui prétendaient avoir étéspoliés debiens par les tyransvenaient plaider devant eux leur cause. II semblequ'àtrès court terme sont apparues à Syracuse des «écoles» où l'on a enseignél'art d'organiser un discours pour convaincre un (interlocuteur-)juge. II est bien évident que cette pratique «démocratique» n'impliquait que les citoyens à part entière. 11. $\mathrm{N}$ e serait-ce qu'en rendant possible la comparaison entre les discours ainsi objectivés, c'est-à-direnon déforméspar lamémoireou lamanipulation qu'impliquelacomparaison. Bien évidemment, cettecomparaison constitue en elle-même un contexte, qui risquait d'être à nouveau méconnu.

12. N otamment dansRhétoriquegénérale(Paris, Larousse, 1970), maisaussi dansR hétoriquedela poésie (Bruxelles, Éd. Complexe, 1977) et jusque dans T raité du signe visuel. Pour une rhétorique de l'image (Paris, Seuil, 1992). 13. Lestenantsdelarhétoriquelinguistiqueprétendent simplement qu'il est possible à un interlocuteur de déduire, à partir d'un état de langue donné, ce qu'un locuteur peut vouloir donner à entendre en disant «fleur» ou «tigresse» quand il entend manifestement parler d'unepersonne. D ansune optique de reproduction du langage, ils sont justifiés de prétendre qu'un interlocuteur peut refaire le cheminement d'un locuteur. $M$ ais cela netient pas dans le concret des interactions où les usages langagiers déjà partagés interviennent uniquement à titre de matière-outil quelecontexte- les deux interlocuteurs - vient informer. $\mathrm{Si}$ « $\mathrm{X}$ » arrive à avoir la même perception d'unechoseet la mêmeviséeà son sujet que « $Y$ », sa vision va nécessairement êtreautre, et cela indépendamment du temps et del'espace, parce qu'elleva en plus se savoir la même.

14. $D$ ans Sémi otique et philosophie du langage, Eco me parait avoir mythifié la question du symbole littéraire en termes de «mode symbolique»: un symbole - au sens littéraire du terme, qui origine des romantiques - serait un signe constitué d'une forme d'expression à laquelle serait liée une nébuleuse de contenu (Paris, P.U .F., 1988, p. 193-238). Je parle de mythe pour la raison suivante: hors contexte, c'est-à-dire quand un discours n'accompagnepasuneinteraction entre desusagers, il y a fort à parier qu'audelà dela subordination du langageà la manifestation d'un dieu immatériel, il est assez facile de circonscrire une nébuleuse de contenu dans un signe le moindrement élaboré - entendre explicitement doublement articulé - ; pour la bonne raison quel'interprétation par l'interlocuteur en fonction du contextedel'échange est toujours elle-mêmeà sanctionner par lelocuteur... Eco continue d'œuvrer dans l'optique de la tradition rhétorique: ce qu'il nomme mode symbolique gagne à être passé au crible de la double articulation asymétrique constitutive des objets langagiers.

15. L'objet du discours du maître, la description des signes, la constitution d'un savoir au sujet du langage, et l'objet du discours pris comme modèle. 16. Entre1904 et 1911, desécolesnormales pour filles ont aussi étéouvertes à Rimouski, Trois-Rivières, N icolet, $\mathrm{H}$ ull, Valleyfield, Joliette et Saint$\mathrm{H}$ yacinthe. Cettemultiplication témoigned'uneproblématiquedelaïcisation du processus de reproduction du langage.

17. Jetiens à souligner ici la qualitédu travail où j'ai puiséà peu près tout ce 
que je sais de la vie de $N$ arcisse $D$ égagné: $R$. D esgagné, «Abbé $N$ arcisse D égagné - 1865-1942 ", sous la rubrique «Littérateurs saguenéens», dans Saguenayensia, vol. 9, n²2, 1967, p. 34-38.

18. En plusdes «N otesdu coursdelittérature», lesarchivesdelacommunauté conservent précieusement plusieurs caisses de manuscrits de D égagné, dont 1) un «C oursdeP sychologieadaptéà l'éducation »dont letextedactylographié fait plus de 130 pages; 2) 17 cahiersmanuscrits où les préparations declasse immédiate côtoient les mots de bienvenue de la rentrée, et où les directives au sujet delaviecouranteau pensionnat voisinent avec des listes derésultats; et 3) quantité de manuscrits de ses publications d'après 1912, soit quelque 53 articlespubliés dansl'Enseignement primaireet environ 300 chroniquesde languepubliées de 1932 à 1940, dans leProgrèsdu Saguenay, sousla rubrique «Q uestions de français». Q uand il devient Principal de l'École normale en 1914, D égagné est déjà un professeur de littérature reconnu. II a publié, entre autres, trois longs articles dans La Revue canadienne. À titre de collaborateur très assidu du journal L'O iseau-mouche du Séminaire, on lui sait une critique sur le vif d'un recueil de N érée Beauchemin, critique qui auraitétéàl'origined'un longsilencedu poète, et plusieursécrits polémiques où il a croiséle fer avec les libéraux, dont Louis F réchette et 0 livar Asselin. 19. Ces renseignementsont étéglanés dansl'article «LesÉcoles normalesde filles de la province de $\mathrm{Q}$ uébec », rédigé par $\mathrm{D}$ égagné lui-même et publié dans L'Enseignement primaire en 1929 (vol. 50, no 9, p. 514-516).

20. Voici un des sujets de composition consignés par ailleurs dans un cahier manuscrit daté de 1912-1913: «U n enfant raconte à sa mère un rêve qu'il a fait pendant la nuit: des anges sont venus et l'ont amenéà Bethléem voir la crèche; au retour, avant de le laisser, ils ont rempli son petit soulier de bonbons». Lesanalyses littéraires porteraient, elles, sur decourts extraits (du Bossuet, plutôt que du Racine ou du Corneille, du moins cette annéelà) et sur des fables, évidemment.

21. C ela ne signifie pas qu'il y a eu 17 séances de cours; le cahier manuscrit - daté de 1912-1913 - de préparation immédiatefait état de 23 séances, de façon très suivie.

22. La partie «C onnaissance des préceptes» ouvre sur les deux autres parties plus explicitement fondées sur la rhétorique; elle se termine sur une leçon intitulée «D u goût», qui fait le lien entre la théorie et les deux «pratiques», la lectureet l'écriture. Cetteleçon sur legoût est elle-mêmeprécédéepar une leçon - dont letitreamalencontreusement étéoublié, lorsdeladactylographie sans doute- qui développe deux idées: 1) «l'art littéraire, la parole littéraire est véritablement un art»; et 2) «la littérature est le premier des arts». O n y apprend également que «L'art littéraire embrasse tous les autres arts; il les contient tous: musique, peinture, sculpture, architecture. Comment les contient-il ? L'art littéraire a sa dernière expression, c'est-à-dire sa plus complète, dans la parole parlée». Et on en vient à savoir ceci : «L'éloquence est l'expression la plus parfaite de l'art littéraire» (p. 24-25).

23. M adame de LaFayette fait partie de sa liste des «maîtres classiques français», mêmesi, sel on lui, le roman est un genreplutôt à déconseiller aux jeunes filles à cause de leur très grande sensibilité. II le leur dit d'ailleurs explicitement, d'autant que c'est dans l'éloquence que se trouve selon lui l'essentiel de l'art littéraire.

24. L'expression «exercice qui consiste à inverser le processus normal de la composition » recoupe parfaitement le postulat fondateur de la tentative du Groupe $\mu$ de généraliser la rhétorique. J'y reviendrai en commentant l'exemple de $D$ égagné déjà annoncé

25. LePetit Robert demeuremoinsaffirmatif : il fait remonter cetteacception à 1611 et en fait état en second, après «qui mérite d'être imité», déjà répertorié au XVIe siècle.

26. Appliquée à l'enseignement del'écrit, cettetradition laisse évidemment tomber les deux phases directement tournées vers la pratique de l'art oratoire, soitlamémorisation du discourset lesrépétitionsoralespréparatoires. $M$ ieux: elle les remplace par la pratique des modèles et les devoirs.

27. À aucun moment, les «N otes» ne font mention de la terminologie grecque ou latine pour désigner les catégories venues de la tradition rhétorique, commec'était l'usage dans les séminai res. Puisqu'il est des écrits deD égagné qui argumentent en faveur de l'importance, dans la formation, del'apprentissage du grec et du latin, j'imagineques'il a prisle soin d'écrire d'avanceson cours, c'est qu'il savait touteladifficultéqu'il relevait, en faisant lepari detransmettresavision deprofesseur deR hétoriqueàdesnormaliennes 28. II n'y est jamais question des procédés rhétoriques précis, ni d'ailleurs nommément de figures de style.

29. O n pourrait imaginer une explication un peu différente: dansl'esprit de la rhétorique classique, l'elocutio s'entend de la mise en forme en vue de I'interlocuteur-juge à convaincre; or, en contexte, l'interlocuteur-juge de l'élève est le professeur même... avec comme conséquence que la mise en formedevient pour ainsi direà adapter au genrepratiquéet peut, au-delà des questions «introduction, développement et conclusion » qui relèvent du sens commun, être entendue en termes de: 1) «savoir faire preuve d'imagination »danslafaçon dedévelopper un sujet pour toucher un interlocuteur : et 2) savoir lier l'expression au contenu.

30. D ansles notes manuscrites des commentairesà fairelors dela remisedes devoirs, toujours danslecahier daté1912-1913, j'ai trouvétracedecequ'est l'équivalent de la narratio dans une analyse littéraire - le plan du texte à analyser - et dans une composition - le fil imaginatif personnel, qui colore et anime la façon de traiter le sujet imposé. M es souvenirs personnels de collège classique viennent ici à la défense de $D$ égagné. $C$ 'est en apprenant l'art de la dissertation que l'on touchait du doigt l'importance de la narration, dela présentation de la manière personnelledont on se proposait de traiter le sujet. Bien évidemment, aucun de mes professeurs n'a insisté pour mefaireremarquer quela «narration »delarhétoriqueétaitl'équivalent de la division du sujet dans une dissertation.

31. Bien sûr, I'avocat pourra toujours regretter sur le tard d'avoir parfois péché... il n'avait pas comme premier devoir d'élever les âmes.

32. Est annexé un exemple d'analyse littéraire de la fable «Le Chêne et le Roseau », fond puis forme, qui propose que le fabuliste excelleà faire passer son âme dans les choses matérielles et partant à nous convaincre

33. On aura beau dire: depuis fort longtemps les littéraires sérieux sont conscients de ce que la littérature est une institution sociale.

34. Q ui lui donne accès à la parole de $D$ ieu.

35. J'insiste. L'invention de l'art oratoire s'est faite à propos d'un passé regretté, postulé idéal par l'ensemble d'une communauté et que cette communauté s'était donné comme «vérité» et comme «bien » à rétablir: à lalumièredes preuvesévoquées par les plaideurs, les jugestranchaient et leur parole devenait reflet du vrai et du bien postulés. Pour D égagné, il y a lieu dene pas confondreart et morale; car il s'agit de subordonner l'un à l'autre: l'art doit servir la morale. Si on n'est pas pour Dieu on est contre: «autrement, il y aurait tout un domaine de choses créées qui échapperaient au C réateur, et leplusimportant, celui des choses intellectuelles [...] Lemot amoral [...] est une absurdité» (p. 21). D 'une part un «objet exprimé» et d'autre part une «âme»; cela implique, dansla perspective de D égagné, que la question du point devue relèveen dernièreinstance de $D$ ieu, auquel peut permettre d'accéder le génie. Ce qui revient à poser l'existence chez les humainsd'une (même) âmeéternellepar rapport àlaquelleD égagnéentend juger, révélation à l'appui, de la plénitude - idéale - des paroles littéraires. À la limite, la théorie de l'âme dont participe Dégagné explique sa compréhension du langage que constitue la tradition rhétorique: celleci postule que la parole d'un interlocuteur-sage reconnu comme juge est synonyme de vérité; et la foi à laquelle adhère $D$ égagnétire la conséquence qui s'impose, en faisant de l'objet exprimé le reflet de $D$ ieu.

36. Ce qui ne signifie pas que la raison accède à $D$ ieu, mais bien qu'elle convient de son existence, en faisant preuve de bon sens devant toutes les merveilles de la création, dont les œuvres d'art.

37. D égagnéne précise pas le prénom; en feuilletant depuis les six volumes des $\mathbb{F}$ uvres complètes de L ouis Racine, je n'ai pas trouvé trace de ces petits vers irréguliers. J'y ai par contre retracé ceci, qui est à tout le moins assez ressemblant: «T el un arbre qu'arrose une onde toujours pure, / 0 rnement 
du rivage, amour de la nature, / Fait espérer les fruits qu'il donne dans leur temps». C'estledébut delasecondestrophed'uneodeintitulée «Lebonheur des] ustes, et lemalheur des M échants». C equi y est dit «T el un arbre[...] 》, c'est celui qui, «plein d'amour et de crainte loin [de Dieu], nuit et jour médite la loi sainte» (L. Racine, $\mathbb{E}$ uvres complètes, t. 2, G enève, Slatkine Reprints, 1969, p. 345). C'est utile de le savoir pour comprendre un peu la spontanéité avec laquelle D égagné identifie ses interlocutrices au jeune lis, dans son commentaire. L'œuvremajeure de Racine-fils, son poème intitulé La Religion, a été reçue comme janséniste au XVIIIe siècle; ce poème didactique était encore pratiqué par les professeurs dans les séminaires du Q uébec au tournant du (XXe) siècle. D égagné connaissait très bien tant ses poèmes que ses réflexions sur la poésie. II ne me paraît pas vain, pour bien entendre le petit quatrain ici en cause, de savoir que dans la préface de son poème-fétiche, Racine-filsa écrit quele plan de son ouvragetient dans cette courte pensée de Pascal : «À ceux qui ont de la répugnance pour la religion, il faut commencer par leur montrer qu'elle n'est pas contraire à la raison, ensuitequ'elleest vénérable; après, la rendreaimable, faire souhaiter qu'elle soit vraie, montrer qu'elle est vraie, et enfin qu'elle est aimable» (L. Racine, La Religion, Lille, L. Lefort, Imprimeur-Libraire, 1853, p. X; jen'ai pas mal transcrit: il s'agit de rendrela religion aimable, puis de montrer qu'ellel'est effectivement - rhétorique oblige!)

38. Si on ne focalise pas de manière univoque sur la pureté des sentiments, on note au passage que Dégagné participe déjà de la vision libérale «expressive» - de l'écriture littéraire: du bien senti d'abord au bien rendu ensuite. Et il reste alors à comprendre ce que la vision libérale doit à la tradition rhétorique, en saisissant pourquoi D égagné ne pouvait que croire au symbolisme «naturel »desromantiquesauxquelsil reprochait- lucidement - d'insister trop sur le sensible. La tradition rhétorique saute ici aux yeux, doublement: d'abord (lefond) «l'idée», puis(laforme) «l'imagesymbolique», quadruple; maisaussi d'abord (l'exordedeD égagné) l'idée, puis(sanarration) l'image symbolique qui permet de tout fondre admirablement dans une même phrase, ensuite (son argumentation) c'est la hauteur de la pensée qui fait la littérature, et finalement (sa péroraison) c'est l'élévation de la pensée - hiérarchie des facultés de l'âme - qui fait le «bien écrit».

39. Cette attribution d'une sentiment à la nature n'est cependant plus très éloignée de l'esprit des textes des romantiques, de même que ceux de Baudelaireet detoutel'écolesymboliste. $D$ égagnépratiquedéjà et pour ainsi direà son insu une lecture romantique - symbole romantiqueà l'appui, j'y arrive- dela petite phrase de Racine. $0 \mathrm{n}$ pourrait en trouver destraces dans des phrases comme celles-ci : «II nefaut pas séparer le corps d'avec l'âme les deux forment un tout complet [sic]. II est vrai que saint Paul a dit: "II y a deux hommes en moi" mais c'est ici la lutte morale [sic]. D ans I'homme physique, il y a unité, harmonie admirable. II y a à ceci des conséquences graves. [...] L'écrivain devraêtreun : ni ange, ni bête, nousnesommesni l'un ni l'autre [sंc] » (p. 4).

40. Si tant est qu'un symbole est un signe arbitraire, mais simplement articulé. En ce sens qu'un même symbole, en chimie par exemple, ne peut pas servir deux fois pour désigner deux objets différents. $\mathrm{O}$ u encore: la balance comme symbole de la justice. On peut savoir que les symboles changent d'une culture à l'autre: le noir renvoie au deuil dans une culture, alors que c'est le blanc qui le fait dans une autre. $M$ ais dans une culture donnée, un symbole a un seul «signifié» possible: contrairement au signe linguistique, il n'est pas doublement articulé.

41. Jeprépareactuellement un essai sur lemythedelaformedanslatradition rhétorique. La thèse que j'y développe est, à gros traits, la suivante: la «forme» d'une brique lui vient du moule; en matière de langage, le moule c'est l'autre, tant pour le locuteur que pour l'interlocuteur.

42. A. M artinet, Élémentsdelinguistiquegénérale, Paris, Collin, 1996, p. 13-15. 